\title{
ESTADO EMOCIONAL EN MUJERES CON CÁNCER DE MAMA: VARIACIÓN EXPERIMENTADA DESPUÉS DE UNA SESIÓN PSICO-ONCOLÓGICA BASADA EN EL COUNSELLING Y LA PSICOLOGÍA POSITIVA
}

\author{
EMOTIONAL STATUS IN WOMEN WITH BREAST CANCER: VARIATION EXPERIENCED \\ AFTER A PSYCHO-ONCOLOGICAL SESSION BASED ON COUNSELING AND \\ POSITIVE PSYCHOLOGY
}

\author{
Lídia Vila Sánchez ${ }^{1}$, Antoni Font Guiteras ${ }^{1}$, Lydia Caba Vela² \\ I Facultad de Psicología, Universidad Autónoma de Barcelona, Barcelona. \\ 2 ICO Girona. Hospital Comarcal Sant Jaume de Calella, Barcelona.
}

Resumen

Objetivo: La mayoría de estudios sobre los beneficios de la intervención psicológica en pacientes de cáncer de mama se han centrado en los efectos acumulados después de varias sesiones terapéuticas. El objetivo de la presente investigación ha sido describir el estado emocional de las pacientes antes de la sesión y constatar el posible efecto emocional inmediato de la misma.

Método: Se evaluó el estado emocional de 30 mujeres diagnosticadas de cáncer de mama, mediante un instrumento ad hoc que incluía ítems del Distress Thermometer for Patients, antes y después de una sesión de apoyo psicológico. Durante la sesión se abordaban aqueIlos aspectos que preocupaban a las pacientes desde un enfoque terapéutico basado en el counselling y la psicología positiva.

Resultados: La media de malestar emocional experimentado durante la semana previa a la sesión era de 6,4 puntos (sobre 10) en el termómetro emocional y más de la mitad de las mujeres habían experimentado varios problemas emocionales durante ese período. Después de la sesión se redujo significativamente la intensidad de las emociones negativas con respecto al inicio de la visita y, también, el malestar emocional en comparación con la última semana. Así mismo, más de la mitad de la
Abstract

Objective: Most studies on psychological intervention benefits have focused on the effects after several therapeutic sessions. The objective of this research was to describe the patients' emotional state, and establish the possible immediate emotional effects of an individual psycho-oncological session.

Method: The emotional state of $\mathbf{3 0}$ women with breast cancer was assessed with an adhoc tool that included items from the Distress Thermometer for Patients at the beginning and at the end of a psychological support session. During the session, issues that worried patients were addressed through an approach based on counselling and positive psychology.

Results: The mean of emotional distress experienced during the previous week was 6.4 on the emotional thermometer, and more than half of the women had experienced multiple emotional problems during this period. After the session, the intensity of certain emotions decreased significantly in relation to the beginning of it, and the emotional distress compared to the previous week also decreased. In addition, more than half the sample experienced positive emotional states. Improvements were related to neither medical condition nor the number of previous psychooncological sessions.

Correspondencia:

Prof. Dr. Antoni Font

Stress and Health Research Group / Grupo de Investigación en Estrés y Salud/ (GIES)

Facultad de Psicología. Edificio B. Campus de la UAB . 08193 Bellaterra (Cerdanyola del Vallès) - Barcelona - Spain

E-mail: Antonio.Font@uab.cat 
muestra experimentaban estados emocionales positivos. Las mejoras no se relacionaron con la situación médica ni con el número de visitas psico-oncológicas previas.

Conclusiones: Ante la constatación de alivio inmediato del malestar emocional, se concluye que es adecuado derivar a todos los pacientes al servicio de psico-oncología al menos para una vista de control.

Palabras clave: Cáncer de mama, intervención psicológica, sesión psico-oncológica, counselling, psicología positiva.
Conclusions: In light of the immediate distress relief, it is concluded that it is appropriate to refer all patients to the psych-oncology service, at least for a psychological assessment visit.

Keywords: Breast cancer, psychological intervention, psycho-oncological session, counselling, positive psychology.

\section{INTRODUCCIÓN}

El cáncer de mama es el más incidente, prevalente y con mayor mortalidad entre las mujeres en España, según datos del $2012^{(1)}$. Se considera una enfermedad crónica que demanda una adaptación psicológica por parte de las pacientes, las cuales están expuestas a situaciones potencialmente muy estresantes durante su diagnóstico, tratamiento, y seguimiento de la enfermedad, lo que puede generar malestar emocional ${ }^{(2-5)}$. La National Comprehensive Cancer Network ${ }^{(6)}$ define el malestar emocional o distress al respecto como una experiencia emocional desagradable, multifactorial, de naturaleza psicológica, social y/o espiritual, que interfiere con la habilidad de afrontar de manera efectiva el cáncer, sus síntomas físicos y su tratamiento. Se extiende a lo largo de un continuo oscilando desde sentimientos normales de vulnerabilidad, tristeza y miedos, hasta problemas que pueden llegar a ser incapacitantes, como depresión, ansiedad, pánico, aislamiento social y crisis existenciales y espirituales. El malestar psicológico en cáncer de mama se daría en aproximadamente un tercio de las pacientes ${ }^{(2)}$, prevaleciendo fundamentalmente los síntomas de ansie- dad y depresión ${ }^{(7)}$. A pesar de ello, cabe señalar que, a la larga, la mayoría de las pacientes se adaptan de forma satisfactoria a su enfermedad ${ }^{(8)}$ e, incluso, algunas experimentan cambios positivos ${ }^{(9)}$, ya sea de forma espontánea o mediante intervención psicológica ${ }^{(10)}$.

Ante este importante impacto emocional, las pacientes que no disponen de suficientes recursos psicológicos y/o psicosociales, pueden beneficiarse de ayuda psico-oncológica desde el momento del diagnóstico ${ }^{(11)}$. Los objetivos de las intervenciones psicológicas suelen ir encaminados a ayudar a los pacientes con cáncer a tolerar mejor el diagnóstico y tratamiento, a disminuir los sentimientos de alienación, aislamiento, indefensión y abandono y, a clarificar percepciones e informaciones erróneas ${ }^{(12)}$, lo que suele traducirse en un incremento de la sensación de control. Generalmente las intervenciones que trabajan con el objetivo de mejorar los recursos de afrontamiento de los pacientes suelen ser de orientación cognitivo-conductual ${ }^{(2)}$. Sin embargo, durante la última década se han desarrollado distintas terapias desde la psicología positiva que han sido utilizadas en mujeres con cáncer de mama con la intención de desarrollar sus fortalezas perso- 
nales, aumentar las emociones positivas, el bienestar, la satisfacción con la vida y el crecimiento y cambio personal ${ }^{(10)}$. Se han propuesto diversas teorías acerca del bienestar psicológico, como por ejemplo, el modelo de las tres vías hacia la felicidad de Seligman (2002), el modelo multidimensional del bienestar psicológico de Ryff (1995) o el modelo motivacional de autodeterminación de Deci y Ryan $(2002)^{(13,14)}$. El mantenimiento de emociones positivas, el compromiso, la puesta en práctica de las fortalezas personales, preservar o recuperar la autonomía, otorgar un sentido a la vida por delante, entre otros, serian elementos que facilitarían el bienestar, pese a la presencia de la enfermedad ${ }^{(15,16)}$. En relación a las emociones, la teoría de la ampliación y construcción de Fredrickson ${ }^{(17)}$ propone que emociones positivas como la alegría, el interés y el amor amplían el repertorio de pensamientos y acciones $y$, como consecuencia, permiten la construcción de nuevos recursos, por lo que podrían participar decisivamente en la adaptación a la enfermedad a largo plazo, además de tener un efecto amortiguador del estrés que suele acompañar a las pacientes, en las diversas fases de la enfermedad ${ }^{(13,16)}$.

Pese a que cada realidad vivencial es subjetivamente única, las mujeres que son diagnosticadas de cáncer de mama suelen pasar por situaciones similares: procedimientos diagnósticos idénticos, espera de resultados, primer tratamiento quirúrgico, etc. Es por esto que, a pesar de las distintas variables que influyen en la vivencia de la enfermedad e, independientemente de la presencia o no de trastornos psicopatológicos, el abordaje psico-oncológico puede ser similar en muchos casos, teniendo en cuenta los aspectos comunes que conlleva padecer esta enfermedad. Esta posición se apoya en el denominado enfoque transdiagnóstico que constata el solapamiento de síntomas y la utilidad y eficacia de las mismas técnicas en situaciones en parte diferentes, proponiendo tratamientos similares o idénticos para diferentes trastornos. Según este enfoque, puede ser útil centrarse en las comunalidades observadas en todos ellos más que en sus diferencias, y tratar aquellos factores subyacentes en todos ellos y no los síntomas específicos ${ }^{(16,18)}$. El counselling, entendido como una forma de relación y comunicación y como un conjunto de destrezas relacionales que, usadas en el proceso interactivo, permiten crear un contexto relacional adecuado ${ }^{(19)}$, podría considerarse también como una estrategia de aplicación transdiagnóstica, ya que utiliza herramientas similares con el objetivo de producir los mismos beneficios en pacientes cuya situación concreta es única(19). En el contexto de padecer un cáncer de mama, las estrategias de intervención breve pueden encaminarse, en primer lugar, al fomento de la autonomía y la reducción de estados emocionales negativos $y$, en segundo lugar, a la facilitación de respuestas positivas, como disfrutar de lo que se tiene o pensamientos de esperanza respecto al futuro.

Para que se dé o se constate un cambio significativo en los aspectos psicosociales generalmente se requiere más de una sesión de intervención psicológica, por lo que la mayoría de estudios se centran en evaluar los efectos de estas terapias después de un número determinado de sesiones (corto plazo) y su mantenimiento a lo largo de un período de seguimiento (largo plazo). No obstante, también es interesante evaluar si en el caso de pacientes que están viviendo una situación potencialmente muy estresante, como el cáncer de mama, es posible constatar alivio emocional inmediato tras la interacción con un profesional de ayuda especializado cuya herramienta de intervención se basa en procedimientos breves como el counselling, teniendo en cuenta la difi- 
cultad de algunas mujeres en seguir una terapia psicológica compleja en determinados momentos de la enfermedad ${ }^{(3)}$, que le obligue a asistir a muchas sesiones a lo largo del tiempo. Por otra parte, la escasez de recursos y el gran volumen de demanda de atención psico-oncológica en algunos casos, obliga a que las intervenciones tengan un formato más breve. La evaluación de los efectos inmediatos se fundamenta en la evaluación ecológica momentánea ${ }^{(20)}$, ya que se tiene en cuenta la experiencia de las pacientes en tiempo real, se constata la variación emocional experimentada íntegramente a lo largo de un período de tiempo objeto de estudio y se reduce, a su vez, el sesgo de memoria presente en otros tipos de evaluación. De hecho, varios estudios realizados en muestras de mujeres con cáncer de mama han utilizado la evaluación ecológica momentánea para evaluar y obtener medidas más precisas sobre la severidad de los síntomas experimentados ${ }^{(20-23)}$. Sin embargo, en el momento de realización de este trabajo no se identificó ningún estudio que tuviera en cuenta los efectos inmediatos producidos a lo largo de una única sesión psico-oncológica. Así pues, los objetivos de la presente investigación han sido, por una parte, conocer el estado emocional de las pacientes con cáncer de mama previo a la visita, concretamente durante la última semana, y justo antes de la misma. Y, por otra, constatar si después de la visita individual con la psico-oncóloga el estado emocional experimenta cambios y con qué posibles variables se relaciona esta variación. Todo ello, con la finalidad última de averiguar la existencia o no de un posible efecto emocional inmediato después de una sola visita, durante la cual las preocupaciones concretas de cada paciente se trabajan desde un mismo enfoque y unos mismos principios englobados en el counselling y la psicología positiva.

\section{MÉTODO}

\section{Muestra}

Las pacientes ambulatorias del Hospital Comarcal Sant Jaume de Calella diagnosticadas de cáncer de mama son derivadas sistemáticamente al Servicio de Psico-oncología desde las Unidades de Oncología, Ginecología y Cirugía. Los criterios de inclusión para participar en el estudio fueron diagnóstico de cáncer de mama como tumor primario, ser mayor de edad y consentir participar en el estudio. Los criterios de exclusión fueron la presencia de deterioro cognitivo que impidiera comprender y/o responder las preguntas, ser menor de edad y haber participado previamente en un estudio psicosocial.

La muestra final estuvo compuesta por un total de 30 mujeres, de edades comprendidas entre los 27 y 83 años (media $=53,6$ y $\mathrm{DT}=13,8)$. La mayoría estaban casadas $(43,3 \%)$ y tenían hijos (80,0\%). En relación a la situación médica en el momento de la valoración, un $23,3 \%$ de las mujeres se encontraban en la fase de diagnóstico, un $46,7 \%$ en la fase de tratamiento y un $30,0 \%$ en la fase de seguimiento. La fase de diagnóstico incluía aquellas mujeres que habían recibido el diagnóstico y, que estaban recibiendo información por parte de los médicos sobre los tratamientos disponibles o que ya estaban esperando iniciarlo. La fase de tratamiento englobaba mujeres que se habían sometido recientemente a una intervención quirúrgica y mujeres que estaban recibiendo quimioterapia o radioterapia, generalmente como tratamiento complementario. Las mujeres en fase de seguimiento eran aquellas que ya habían finalizado el tratamiento inicial, aunque recibieran hormonoterapia preventiva, y que acudían al hospital para las revisiones médicas periódicas. En cuanto al número de visita psicológica, en un $60 \%$ de los casos se trataba de la primera vez que la paciente era visitada por la psico-oncóloga (tabla 1). 


\section{Tabla 1. Características demográficas y médicas de las pacientes $(\mathbf{N}=\mathbf{3 0})$}

\begin{tabular}{|c|l|r|r|}
\hline \multirow{2}{*}{ Edad (años) } & Media (D.T) 53,6 (13,8) \\
& Rango 27-83 & $\mathrm{n}$ & \multicolumn{1}{c|}{$\%$} \\
\hline & & 2 & $6,7 \%$ \\
& Soltera & 1 & $3,3 \%$ \\
& Pareja sin convivencia & 6 & $20,0 \%$ \\
Estado Civil & Pareja con convivencia & 13 & $43,3 \%$ \\
& Casada & 5 & $16,7 \%$ \\
& Separada/divorciada & 3 & $10,0 \%$ \\
\hline \multirow{2}{*}{ Hijos } & Viuda & 6 & $20,0 \%$ \\
& No & 24 & $80,0 \%$ \\
\hline \multirow{2}{*}{ Número de visita } & Sí & 18 & $60,0 \%$ \\
& Primera visita & 12 & $40,0 \%$ \\
\hline \multirow{3}{*}{ Situación médica actual } & Visita sucesiva & 7 & $23,3 \%$ \\
& Diagnóstico & 14 & $46,7 \%$ \\
& Tratamiento & 9 & $30,0 \%$ \\
\hline
\end{tabular}

Instrumentos y variables analizadas

Existen varios instrumentos para evaluar aspectos psicosociales en pacientes con cáncer que tienen un formato breve y que son relativamente fáciles de aplicar como el de Detección de Malestar Emocional en enfermos al final de la vida ${ }^{(24)}$. Distintos estudios realizados en pacientes con cáncer de mama han utilizado escalas visuales análogas ${ }^{(11,25-27)}$. Teniendo en cuenta los objetivos y las características del presente estudio, se debía disponer de un instrumento muy sencillo y fácil de administrar para poder ser completado en la misma visita, que aportara información del estado emocional, antes de empezar y al acabar la visita, ajustándose a las limitaciones temporales y de espacio de éstas. También debía ser un instrumento fácilmente comprensible, lo menos intrusivo posible y que los ítems no indujeran por si solos a un determinado estado emocional. Además, se debían evaluar aspectos susceptibles de experimentar variación durante la visita psico-oncológica. Así pues, optamos por utilizar un sistema de valoración mediante ítems en forma de escala visual analógica adaptados del Distress Thermometer for Patients, un sistema ampliamente utilizado y recomendado por la National Comprehensive Cancer Network para la detección de malestar emocional en pacientes oncológicos $^{(11,28-34)}$. El instrumento aplicado constaba de dos versiones, una que se administraba antes de la visita y otra después. Al inicio se evaluaba: 1) malestar emocional experimentado la última semana (indicar el número que mejor definiera su malestar en un termómetro que iba del 0, mínimo, al 10, máximo); 2) si la persona había sufrido problemas emocionales como depresión, miedo, nerviosismo, tristeza, preocupación y pérdida de interés en las actividades cotidianas durante la última semana (marcar sí o no); 3) intensidad de tristeza, rabia, miedo y nerviosismo en el momento actual (marcar el número que mejor definiera su estado en una recta numérica que oscilaba del 0, mínimo, al 10, máximo). Después de la sesión se evaluaba, debiendo responder según el momento actual: 1) el males- 
tar emocional que se experimentaba; 2) si la persona se sentía animada, valiente, tranquila, alegre, despreocupada y con interés por las actividades; 3 ) intensidad de tristeza, rabia, miedo y nerviosismo. El formato era el mismo que la primera parte del instrumento. Los ítems sobre la intensidad de las emociones negativas antes y después de la visita están basados en propuestas sobre lo que la evaluación del malestar emocional debería incluir ${ }^{(35)}$ y que generalmente evalúan instrumentos más complejos utilizados en pacientes con cáncer ${ }^{2,24,30)}$.

Además de las variables correspondientes a cada uno de los ítems comentados, se generaron tres nuevas variables. La primera de ellas, denominada "alteración emocional antes de la visita" fue obtenida a partir de la suma de la intensidad de tristeza, rabia, miedo y nerviosismo antes de la visita. La segunda, "alteración emocional después de la visita" era la suma de la intensidad de estas mismas emociones después de la visita. Finalmente, la variable "cambio emocional" representaba la diferencia entre las dos anteriores. Así pues, las puntuaciones en "alteración emocional" podían oscilar entre 0 y 40 y en "cambio emocional" entre -40 y 40 .

\section{Procedimiento}

Las pacientes que acudieron a la visita con la psico-oncóloga entre mayo del 2015 y enero del 2016 eran informadas al inicio, sobre el objetivo y procedimiento del estudio. En caso de que decidieran participar voluntariamente firmaban el consentimiento informado $y$, seguidamente, se les administraba la parte del instrumento referente al estado emocional en la última semana y en el momento presente, antes de la visita. Seguidamente tenía lugar la interacción terapéutica entre la paciente y la psico-oncóloga. Una vez finalizada la visita, se administraba la última parte del instrumento en relación a su estado emocional en ese momento, después de la sesión. Ninguna de las pacientes rechazó colaborar con la investigación.

\section{Desarrollo de la sesión}

Las sesiones tenían lugar de forma individual. El enfoque terapéutico presente en todas ellas era el counselling. Mediante una comunicación caracterizada fundamentalmente por la escucha activa, la empatía y la congruencia por parte de la psico-oncóloga, se pretendía crear un contexto emocional y relacional adecuado, donde la persona se sintiera tranquila, escuchada, entendida y apoyada, además de no juzgada y libre de expresar todo aquello que deseara (temores, deseos y necesidades). Se daba especial importancia al vínculo terapéutico basado en la igualdad, la confianza y la cercanía entre la paciente y la psico-oncóloga, respetando los límites de ambos roles. Se fomentaba una actitud proactiva de la paciente a lo largo de todo el proceso, de manera que era la paciente la que marcaba el ritmo de la intervención según su evolución. En el caso de que se tratara de una primera entrevista se informaba a la paciente que las sesiones serian un espacio para hablar de todo aquello que deseara o le preocupara, no solamente sobre la enfermedad. Las sesiones trascurrían de forma flexible y no directiva, centrándose en aquellos temas que más interesaban o preocupaban a cada paciente según la situación en la que se encontraban en los diferentes momentos y ámbitos de su vida.

Todas las sesiones seguían una estructura similar: en primer lugar las pacientes relataban su vivencia problemática; seguidamente, la psico-oncóloga sugería una nueva perspectiva del problema y una manera más adaptativa de afrontar 
la situación, proponiendo distintas herramientas y estrategias que a continuación se discutían con la paciente; para acabar, se resolvían dudas y se pactaba como tareas para casa el poner en práctica los distintos aspectos comentados y trabajados. El objetivo perseguido a lo largo de las sesiones era mejorar el bienestar y los aspectos alterados de la calidad de vida de las mujeres, acompañándolas durante el proceso de la enfermedad, aumentando su sensación de control y cultivando sus fortalezas personales, cogniciones, conductas y emociones positivas. Se aplicaron las siguientes técnicas o estrategias, en función de las necesidades predominantes de cada paciente:

a) Resolución de problemas. Se identificaban aquél o aquellos problemas que generaban más malestar en la paciente y se intentaban generar, conjuntamente, soluciones alternativas y proactivas, basadas en el aquí y ahora y en los recursos de cada paciente. Se remarcaba la importancia de centrarse en las posibilidades y no en los impedimentos.

b) Psicoeducación. Se facilitaba información y consejos prácticos sobre distintos aspectos del proceso de enfermedad. Por ejemplo, asesoramiento sobre los efectos secundarios del tratamiento (molestias postoperatorias, alopecia, etc.), ayuda en la reorganización de tareas y obligaciones durante el tratamiento de radioterapia (requería desplazarse diariamente a un hospital de referencia ubicado en otra comarca), etc. Con una información adecuada se perseguía: disminuir la impredecibilidad, la ansiedad anticipatoria y la ansiedad concurrente al tratamiento; $y$, aumentar la autonomía, los autocuidados, la seguridad y la autocon- fianza de la persona enferma que, en ese momento, podía tener poca sensación de control sobre su vida.

c) Restructuración cognitiva. Se ayudaba a las pacientes a identificar pensamientos negativos, a darse cuenta de cómo influían en su estado de ánimo y en su conducta, a examinar su evidencia a favor y en contra $y$, seguidamente, a sustituirlos por otros más positivos. A partir de este proceso se modificaban concepciones erróneas entorno al cáncer y se intentaban disminuir, por ejemplo, sentimientos de culpabilidad e impotencia. Se les proponía enfocar el pensamiento hacia aspectos positivos que proporcionaran bienestar y, así, fomentar el optimismo y la esperanza, todo ello con la finalidad de facilitar una mejor adaptación a la enfermedad.

d) Atención plena/mindfulness. Se fomentaba la autoobservación y la aceptación de los pensamientos y sentimientos, fueran positivos o negativos sin enjuiciarlos. Se insistía a las pacientes que se mantuvieran, mentalmente, en el momento presente, lo único de lo que disponían. El pasado ya no existía y, el futuro, nadie les podía asegurar como sería. Habían recibido un diagnóstico, pero el pronóstico formaba parte del futuro y anticiparlo solo generaba angustia. Para facilitarles estar presentes en el aquí y ahora, en la primera sesión se les enseñaba una sencilla y breve relajación con las siguientes indicaciones: centrar la atención en la respiración, concentrarse en cómo es la inspiración y la expiración, permitirse disfrutar de las sensaciones que se sienten y dejar pasar los pensamientos que aparecen. El ejercicio duraba unos dos o tres 
minutos y se les aconsejaba que la pusieran en práctica cada día, como mínimo al despertarse y al acostarse, y siempre que lo desearan. Se destacaba que era un remedio que tenían disponibles las 24 horas del día, gratuito, sin efectos secundarios y de mayor eficacia cuanto más lo usaran.

e) Activación conductual. Se aconsejaba a las pacientes que realizaran algún tipo de actividad física, que recuperaran o continuaran con aquellas actividades que les resultaban placenteras, que no estuvieran todo el día en casa y que mantuvieran cierto contacto social, siempre dentro de sus posibilidades.

f) Visualización. La terapeuta proponía combinar la relajación con la programación o visualización de cómo quería la paciente que fuera su día o cualquier aspecto de su vida. Por ejemplo, en el caso de una mujer que iba a ser intervenida quirúrgicamente, se le decía que durante la respiración podía visualizar como quería que fuera la intervención, siempre desde una perspectiva positiva (estaría tranquila antes de entrar al quirófano, la recuperación sería rápida y sin dolores, etc.).

g) Desarrollo de aspectos positivos. Incluía elementos como los siguientes. Considerar la enfermedad como una oportunidad y proceso de crecimiento personal, aceptando las limitaciones que ésta puede conllevar. Reconocer y pensar en sus fortalezas personales y aplicarlas en la vida cotidiana. Permitirse el desahogo emocional pero posteriormente utilizar la relajación para canalizar y reorientar de nuevo el pensamiento hacia aspectos positivos. Marcarse objetivos positivos y alcanzables, aceptando un posible sentido en todo aquello que hacían. Fomentar el agradecimiento y el perdón: perdonar no implicaba justificar acciones impropias, sino dejar de sentir rabia, culpabilidad, frustración, etc., para sentir alivio y libertad. Por último, también se aconsejaba no perder el sentido del humor.

\section{Diseño y análisis de datos}

Según la clasificación de las metodologías de investigación en psicología propuesta por Montero y León ${ }^{(36)}$, el presente trabajo se engloba dentro del grupo de estudios empíricos cuantitativos y descriptivos, con medidas secuenciales intrasujeto, pre y post tratamiento. El análisis de datos se realizó con el programa estadístico SPSS Statistics 21. La descripción del estado emocional de las pacientes en los diferentes momentos temporales se indica mediante frecuencias absolutas $y$ porcentajes en el caso de las variables categóricas y, con medias y desviaciones típicas cuando se trata de variables cuantitativas. Para el análisis comparativo y correlacional se han utilizado estadísticos no paramétricos, que por un lado, en el caso de comparaciones entre-condiciones se ajustan al número reducido de sujetos pertenecientes a cada una y, por otro, no implican restricciones acerca de las características de la variable en la población, puesto que mediante la prueba Shapiro-Wilk se comprobó que las variables no seguían una distribución normal en la población. Para comparar el malestar emocional (medido a través del termómetro emocional), el total de problemas emocionales durante la última semana, la tristeza, rabia, miedo, nerviosismo y alteración emocional antes de la visita, y el cambio emocional experimentado después de la visita, en función de la si- 
tuación médica y del número de visita, se aplicaron las pruebas de Kruskal-Wallis y $U$ de Mann-Whitney respectivamente. Para valorar el efecto emocional de la sesión se emplearon las pruebas de Wilcoxon y McNemar. La primera de ellas se utilizó para comparar la intensidad de las diferentes emociones antes y después de la visita, la alteración emocional entre estos dos momentos y, el malestar emocional y el total de problemas emocionales entre la última semana y después de la visita. La prueba de McNemar fue usada específicamente para determinar si después de la intervención psico-oncológica el cambio experimentado en los estados emocionales respecto a la última semana eran o no estadísticamente significativos. Finalmente, para el análisis correlacional se ha calculado el coeficiente de correlación de Spearman. Éste abarcó las asociaciones entre: la intensidad de las diferentes emociones antes de la visita; la intensidad de les emociones después de la sesión; el malestar emocional experimentado después de la visita y la intensidad de las emociones en ese mismo momento; el malestar emocional de la última semana y la intensidad de las emociones y, alteración global, antes de la visita; y, el malestar emocional de la última semana y el de después de la visita. En todas las pruebas se ha empleado un nivel de confianza del 95\%.

\section{RESULTADOS}

1. Estado emocional durante la última semana

La media de malestar emocional experimentado la última semana, medido a partir del termómetro emocional, es de $6,4(\mathrm{DT}=3,3)$. En la tabla 2 se indica la frecuencia y el porcentaje de mujeres que han experimentado los distintos problemas emocionales. Los más frecuentes son preocupación (en el 90,0\% de los casos), nerviosismo $(76,7 \%)$, tristeza $(73,3 \%)$, depresión $(60,0 \%)$ y miedo $(53,3 \%)$, seguidos de la pérdida de interés en las actividades cotidianas (43,3\%). Todas las pacientes experimentaron al menos uno de los problemas indicados, predominando la presencia de 4 o 6 problemas conjuntamente (el $23,3 \%$ de las pacientes en ambos casos).

No se observó ninguna relación significativa entre el malestar emocional experimentado y la situación médica actual, es decir, el malestar emocional no difiere significativamente entre aquellas mujeres que se encuentran en la fase de diagnóstico, de tratamiento o de seguimiento. Tampoco se observa relación entre éste y el que se trate o no de la primera visita psico-oncológica. Sucede lo mismo con el total de problemas emocionales, tanto en relación a la situación médica actual, como en relación a si se trataba o no de primeras visitas (ver tablas 3 y 4 , respectivamente).

Tabla 2. Frecuencia y porcentaje de mujeres que han experimentado cada problema emocional durante la última semana previa a la visita psico-oncológica

\begin{tabular}{|l|c|c|}
\hline Problema emocional & $\mathbf{n}$ & $\mathbf{\%}$ \\
\hline Preocupación & 27 & 90,0 \\
\hline Nerviosismo & 23 & 76,7 \\
\hline Tristeza & 22 & 73,3 \\
\hline Depresión & 18 & 60,0 \\
\hline Miedo & 16 & 53,3 \\
\hline Pérdida de interés por las actividades cotidianas & 13 & 43,3 \\
\hline
\end{tabular}




\section{Tabla 3. Puntuaciones medias (y desviaciones típicas) en malestar emocional de mujeres que se encuentran en diferentes fases de la enfermedad}

\begin{tabular}{|l|c|c|c|}
\hline & Diagnóstico & Tratamiento & Seguimiento \\
\hline \multirow{2}{*}{ Malestar emocional última semana } & $\begin{array}{c}6,4 \\
(3,7)\end{array}$ & $\begin{array}{c}6,1 \\
(3,9)\end{array}$ & $\begin{array}{c}6,8 \\
(2,0)\end{array}$ \\
\hline \multirow{2}{*}{ Total problemas emocionales última semana } & 4,0 & 4,1 & 3,7 \\
& $(1,6)$ & $(1,8)$ & $(1,4)$ \\
\hline \multirow{2}{*}{ Tristeza antes visita } & 4,7 & 4,1 & 5,6 \\
& $(4,1)$ & $(3,5)$ & $(2,7)$ \\
\hline \multirow{2}{*}{ Rabia antes visita } & 7,1 & 2,8 & 3,2 \\
& $(4,9)$ & $(3,7)$ & $(4,0)$ \\
\hline \multirow{2}{*}{ Miedo antes visita } & 4,7 & 3,6 & 3,9 \\
& $(4,0)$ & $(3,6)$ & $(3,7)$ \\
\hline \multirow{2}{*}{ Nerviosismo antes visita } & 6,1 & 4,5 & 5,7 \\
& $(3,8)$ & $(4,1)$ & $(3,6)$ \\
\hline \multirow{2}{*}{ Alteración emocional antes visita } & 22,7 & 14,9 & 18,3 \\
& $(13,1)$ & $(11,7)$ & $(11,5)$ \\
\hline \multirow{2}{*}{ Cambio emocional } & 1,7 & 6,6 & 9,9 \\
& $(16,4)$ & $(7,0)$ & $(9,0)$ \\
\hline
\end{tabular}

Nota: La comparación de los grupos mediante la prueba de Kruskal-Wallis no aportó resultados estadísticamente significativos entre los grupos ( $p<0,05$, bilateral).

Tabla 4. Puntuaciones medias (y desviaciones típicas) en malestar emocional de mujeres que acuden a una primera visita psico-oncológica o a una visita posterior

\begin{tabular}{|l|c|c|}
\hline & Primera visita & Visita posterior \\
\hline Malestar emocional última semana & 6,1 & 6,8 \\
& $(3,9)$ & $(2,0)$ \\
\hline \multirow{2}{*}{ Total problemas emocionales última semana } & 4,2 & 3,7 \\
& $(1,8)$ & $(1,3)$ \\
\hline \multirow{2}{*}{ Tristeza antes visita } & 4,6 & 4,8 \\
& $(3,8)$ & $(2,9)$ \\
\hline \multirow{2}{*}{ Rabia antes visita } & 5,3 & 1,9 \\
& $(4,7)$ & $(2,8)$ \\
\hline \multirow{2}{*}{ Miedo antes visita } & 4,2 & 3,5 \\
& $(3,7)$ & 5,6 \\
\hline \multirow{2}{*}{ Nerviosismo antes visita } & 5,0 & $(3,1)$ \\
\hline \multirow{2}{*}{ Alteración emocional antes visita } & $(4,4)$ & 15,8 \\
& 19,1 & $7,4)$ \\
\hline \multirow{2}{*}{ Cambio emocional } & $(13,9)$ & $(7,9)$ \\
\hline
\end{tabular}

Nota: La comparación de grupos mediante la prueba de $U$ de Mann-Whitney no aportó resultados estadísticamente significativos $(p<0,05$, bilateral).

2. Estado emocional antes de la visita

Antes de la visita la emoción negativa más intensa indicada es el nerviosismo (media $=5,2$ ), seguido de la tristeza (me$d i a=4,7)$, la rabia (media $=3,9)$ y el miedo (media $=3,9)$, tal y como se detalla en la tabla 5. En la matriz de correlaciones entre 
estas cuatro emociones (tabla 6) se observa que todas ellas correlacionan directa y significativamente entre sí. Las más significativas $(p=0,01)$ son las correlaciones entre el miedo y las demás emociones (tristeza, rabia y nerviosismo). La media de alteración emocional es 17,8 puntos (DT=11,9).

Nuevamente, la intensidad de las emociones de tristeza, rabia, miedo y nerviosismo y, la alteración emocional, no guardan relación con la situación médica en que se encuentran las mujeres (tabla 3). Así mismo, las pacientes que acuden a una primera visita no se diferencian emocionalmente de las mujeres que ya han tenido alguna o algunas visitas previas (tabla 4). Sin embargo, como puede observarse en la tabla 4, las medias en la intensidad de rabia, miedo, nerviosismo y alteración emocional, son ligeramente superiores en la fase de diagnóstico.

\section{Estado emocional después de la visita}

Como se puede observar en la tabla 7, después de la visita la emoción más intensa pasa a ser la rabia (media $=3,1)$, seguida por el nerviosismo (media $=3,0$ ), la tristeza (media $=2,8$ ) y el miedo (media $=2,5$ ). A partir de la matriz de correlaciones (tabla 8), se constata como existe una correlación positiva y significativa entre todas estas emociones $(p=0,01)$. La media de alteración emocional es 11,3 puntos (DT=11,9). Por otro lado, la media de malestar experimentado después de la visita (puntuaciones del termómetro emocional) es ahora de 2,7 puntos $(\mathrm{DT}=3,1)$ y correlaciona directa y significativamente con la intensidad de la tristeza $(\rho=0,729 ; p=0,01)$, la rabia $(\rho=0,435$; $p=0,05)$, el miedo $(\rho=0,664 ; p=0,01)$, el nerviosismo $(\rho=0,640 ; p=0,01)$ y la alteración emocional $(\rho=0,704 ; p=0,01)$, observada en este momento.

Respecto a las emociones positivas, como se indica en la tabla 9, después de la visita la mayoría de las mujeres se sienten animadas $(83,3 \%)$, valientes $(83,3 \%)$, con interés por las actividades cotidianas $(83,3 \%)$, tranquilas $(63,3 \%)$ y alegres $(63,3 \%)$. Solo en el caso de la preocupa-

\section{Tabla 5. Valores medios y desviaciones típicas de la intensidad de las emociones antes de la visita}

\begin{tabular}{|l|c|c|}
\hline Emoción & Media & Dev.std \\
\hline Nerviosismo & 5,2 & 3,8 \\
\hline Tristeza & 4,7 & 3,4 \\
\hline Rabia & 3,9 & 4,3 \\
\hline Miedo & 3,9 & 3,7 \\
\hline
\end{tabular}

Tabla 6. Correlaciones Spearman entre las emociones antes de la visita

\begin{tabular}{|l|r|r|r|r|}
\hline Emoción & \multicolumn{1}{|c|}{ Tristeza } & \multicolumn{1}{|c|}{ Rabia } & \multicolumn{1}{c|}{ Miedo } & Nerviosismo \\
\hline Tristeza & 1 & 1 & & \\
\hline Rabia &, $458^{*}$ & $\mathbf{, 5 5 4}^{* *}$ & 1 & \\
\hline Miedo & $\mathbf{4 7 4}^{* *}$ &, $362^{*}$ &, $\mathbf{7 0 8}^{* *}$ & 1 \\
\hline Nerviosismo &, $447^{*}$ & & & \\
\hline
\end{tabular}

**La correlación es significativa al nivel de $p<0,01$ (bilateral).

*La correlación es significativa al nivel de $p<0,05$ (bilateral).

En negrita las correlaciones más elevadas. 


\section{Tabla 7. Valores medios y desviaciones típicas de la intensidad de las emociones después de la visita}

\begin{tabular}{|l|c|c|}
\hline Emoción & Media & Dev.std \\
\hline Rabia & 3,1 & 4,0 \\
\hline Nerviosismo & 3,0 & 3,5 \\
\hline Tristeza & 2,8 & 2,7 \\
\hline Miedo & 2,5 & 3,6 \\
\hline
\end{tabular}

Tabla 8. Correlaciones Spearman entre las emociones después de la visita

\begin{tabular}{|l|r|r|r|r|}
\hline Emoción & \multicolumn{1}{|c|}{ Tristeza } & \multicolumn{1}{|c|}{ Rabia } & \multicolumn{1}{|c|}{ Miedo } & Nerviosismo \\
\hline Tristeza & 1 & 1 & & \\
\hline Rabia & $\mathbf{5 1 1}^{* *}$ & $\mathbf{5 3 1}^{* *}$ & 1 & \\
\hline Miedo & $\mathbf{7 1 1}^{* *}$ & $\mathbf{, 6 8 5}^{* *}$ & $\mathbf{7 9 2}^{* *}$ & 1 \\
\hline Nerviosismo &, $\mathbf{6 5 7}^{* *}$ & & & \\
\hline
\end{tabular}

**La correlación es significativa al nivel de $p<0,01$ (bilateral)

*La correlación es significativa al nivel de $\mathrm{p}<0,05$ (bilateral)

ción, el porcentaje de mujeres que se sienten preocupadas después de la visita supera al de mujeres que se sienten despreocupadas (60,0 y $40,0 \%$ respectivamente). La tabla 10 muestra la frecuencia y porcentaje de mujeres según el total de estos estados emocionales positivos. Se observa como la mayoría de mujeres experimentan entre 4 y 6 estados emocionales positivos conjuntamente $(26,7 \%$ de las pacientes en cada caso). Solo en un caso no apareció ningún estado emocional positivo después de la visita.
4. Relación entre el estado emocional de la última semana y el de antes de la visita

La puntuación de malestar emocional experimentado la última semana correlacionó significativamente con la intensidad de tristeza $(\rho=0,569 ; p=0,01)$ y la alteración emocional de antes de la visita $(\rho=, 414 ; p=0,05)$. No obstante, no se observó ninguna asociación significativa entre el malestar emocional de la última semana y la intensidad del resto de emociones negativas antes de la visita.

\section{Tabla 9. Frecuencia y porcentaje de mujeres que experimentan cada estado emocional positivo después de la visita}

\begin{tabular}{|l|c|c|}
\hline Estado emocional positivo & $\mathbf{n}$ & $\mathbf{\%}$ \\
\hline Ánimo & 25 & 83,3 \\
\hline Valentía & 25 & 83,3 \\
\hline Interés en las actividades cotidianas & 25 & 83,3 \\
\hline Tranquilidad & 19 & 63,3 \\
\hline Alegría & 19 & 63,3 \\
\hline Despreocupación & 12 & 40,0 \\
\hline
\end{tabular}




\section{Tabla 10. Frecuencia y porcentaje de mujeres según el total de estados emocionales positivos experimentados después de la visita}

\begin{tabular}{|c|c|c|}
\hline Estados emocionales positivos & $\mathbf{n}$ & $\mathbf{\%}$ \\
\hline 0 & 1 & 3,3 \\
\hline 1 & 0 & 0,0 \\
\hline 2 & 4 & 13,3 \\
\hline 3 & 4 & 13,3 \\
\hline 4 & 8 & 26,7 \\
\hline 5 & 5 & 16,7 \\
\hline 6 & 8 & 26,7 \\
\hline
\end{tabular}

5. Comparación del estado emocional antes de la visita y después de ésta

Los resultados del análisis comparativo de las emociones negativas indican que la intensidad de tristeza $(Z=-3,239$; $\mathrm{p}=0,001)$, miedo $(Z=-2,416 ; p=0,016)$ y nerviosismo $(Z=-3,030 ; p=0,002)$ y, la alteración emocional $(Z=-3,475 ; p=0,001)$, después de la visita, son significativamente inferiores que antes de ésta. En el caso de la rabia, a pesar de constatarse una disminución, ésta no es significativa. Las puntuaciones medias y desviaciones típi- cas obtenidas antes y después de la visita se indican en la tabla 11 y, la representación gráfica de la disminución de las cuatro emociones en la figura 1.

Si consideramos el cambio emocional experimentado (media $=6,4$; DT=10,41), es decir, la diferencia entre la alteración emocional antes de la visita y la alteración emocional después de ésta, éste no se relaciona significativamente con la situación médica actual ni con el número de visita. No obstante, las medias obtenidas según la situación médica actual (tabla 3 ) indican mayor cambio en aque-

Tabla 11. Puntuaciones medias (y desviaciones típicas) en intensidad de nerviosismo, tristeza, rabia, miedo y alteración emocional conjunta, antes y después de la visita

\begin{tabular}{|l|c|c|}
\hline & Antes visita & Después visita \\
\hline \multirow{2}{*}{ Nerviosismo } & 5,2 & $3,0^{*}$ \\
\multirow{2}{*}{ Tristeza } & $(3,8)$ & $(3,5)$ \\
\hline \multirow{2}{*}{ Rabia } & 4,7 & $2,8^{*}$ \\
& $(3,4)$ & $(2,7)$ \\
\hline \multirow{2}{*}{ Miedo } & 3,9 & 3,1 \\
& $(4,3)$ & $(4,0)$ \\
\hline \multirow{2}{*}{ Alteración emocional } & 3,9 & $2,5^{*}$ \\
& $(3,7)$ & $(3,6)$ \\
\hline
\end{tabular}

*Las comparaciones intra-condición mediante la prueba de Wilcoxon son estadísticamente significativas al nivel de $\mathrm{p}<0,05$ (bilateral). 
Figura 1. Valores medios de intensidad de tristeza, rabia, miedo y nerviosismo antes y después de la visita

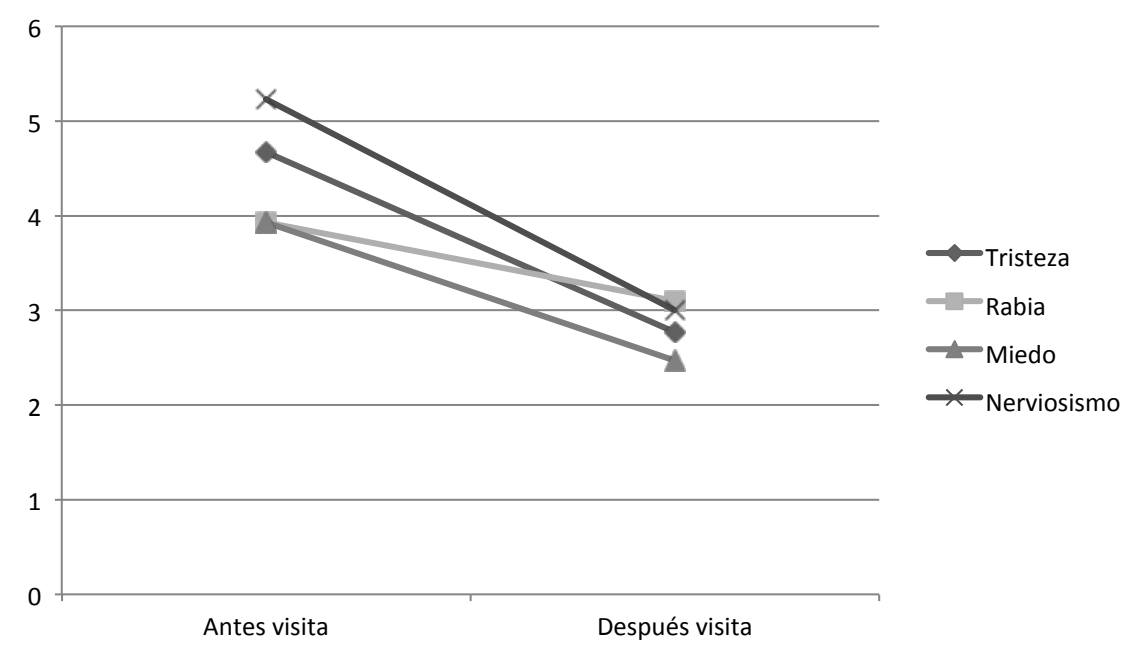

Ilas mujeres que se encuentran en fase seguimiento médico. Respecto a la visita psico-oncológica, la media de cambio es menor entre las mujeres que acuden a una primera visita (tabla 4).

\section{Comparación del estado emocional de} la última semana y después de la visita

Si se compara el estado emocional de la última semana con el observado después de la visita psicológica se obtienen los siguientes resultados. En primer lugar, existe una correlación directa y significativa entre el malestar emocional experimentado en ambos momentos temporales $(\rho=0,488 ; p=0,01)$. En segundo lugar, mediante la prueba de Wilcoxon se constata que el malestar experimentado después de la visita es significativamente inferior que el experimentado a lo largo de la última semana $(Z=-4,149$; $p<0,001)$. Esta misma prueba también indica que durante la última semana se han experimentado más problemas emo- cionales que los observados al final de la visita $(Z=-3,881 ; p<0,001)$, pese a que la paciente se encuentra en un medio hospitalario. Las puntuaciones medias y desviaciones típicas de ambas variables, obtenidas la última semana y después de la visita, se indican en la tabla 12. Finalmente, la prueba de McNemar indica que cuando hay un cambio de polo en el estado emocional experimentado entre la última semana y después de la visita, es más probable que este cambio sé de en el sentido de negativo a positivo y no al revés. Esto es así en todos los estados emocionales considerados: un $77,8 \%$ de mujeres pasan de sentirse deprimidas a sentirse animadas $(p=0,001)$, un $81,3 \%$ de sentir miedo a sentirse valientes ( $p=0,007$ ), un $60,9 \%$ del nerviosismo a la tranquilidad $(p=0,004)$, un $54,5 \%$ de la tristeza a la alegría $(p=0,003)$, un $37,0 \%$ de sentirse preocupadas a la despreocupación $(p=0,012) y$, un $61,5 \%$ consiguen recuperar el interés en las actividades cotidianas $(p=0,008)$. 


\section{Tabla 12. Puntuaciones medias (y desviaciones típicas) en malestar emocional y total de problemas emocionales la última semana y después de la visita}

\begin{tabular}{|l|c|c|}
\hline & Última semana & Después visita \\
\hline Malestar emocional & 6,4 & $2,7^{*}$ \\
& $(3,3)$ & $(3,1)$ \\
\hline Total problemas emocionales & 4,0 & $1,8^{*}$ \\
& $(1,6)$ & $(1,6)$ \\
\hline
\end{tabular}

*Las comparaciones intra-condición mediante la prueba de Wilcoxon son estadísticamente significativas al nivel de $p<0,05$ (bilateral)

\section{DISCUSIÓN}

Los objetivos de la presente investigación han sido, por un lado, analizar el estado emocional de una muestra de mujeres con cáncer de mama durante la última semana y cuando se encuentran en el centro sanitario, justo antes de la visita psico-oncológica. Por otro lado, se ha querido constatar si las pacientes experimentan variación en su estado de ánimo después de la sesión psicológica y si hay diferencias en función de la fase de enfermedad en que se encuentran las pacientes y de si se trata o no de la primera visita con la psico-oncóloga. No se pretendía conocer los efectos de una terapia una vez finalizada, sino constatar si es posible cambiar la dinámica emocional de las pacientes en una sola sesión a partir de una interacción terapéutica individual basada en el counselling y la psicología positiva. Esta orientación, común en todos los casos, guiaba, acompañaba y englobaba las distintas intervenciones terapéuticas que se aplicaban y adaptaban a cada caso concreto. La evaluación basada en el momento presente, tanto antes como después de la visita, ofrece las ventajas propias de la evaluación ecológica momentánea, sobretodo la reducción del sesgo de memoria y el poder constatar esta variación emocional experimentada justo después de la sesión psico-oncológica ${ }^{(20)}$, antes de que intervengan otros factores.
Los resultados obtenidos sugieren que la mayoría de pacientes experimentan malestar emocional durante la última semana, previa a la visita psico-oncológica. Una puntuación igual o mayor a 4 en el termómetro emocional indica que la paciente presenta un malestar emocional moderado-severo $^{(6)} y$, en el presente caso, la media se sitúa en una puntuación de 6,4, muy superior por ejemplo a la obtenida en el estudio de Lester et al. ${ }^{(28)}$ (media $=4,3$ ) en una muestra, también, de mujeres con cáncer de mama en distintas fases de la enfermedad. Al menos tres cuartas partes de la muestra han experimentado durante la última semana preocupación y nerviosismo y, más de la mitad, tristeza, depresión y miedo. La reacción menos frecuente es la pérdida de interés en las actividades cotidianas. Llama la atención que los valores de afectación en nuestra muestra son mayores que los observados en el mismo estudio de Lester et al. ${ }^{(28)}$, si bien se coincide en que las emociones negativas más frecuentes también son la preocupación, el nerviosismo y la tristeza.

Si comparamos el estado emocional de la última semana con el de después de la visita se constata una disminución significativa en el nivel de malestar, de manera que la puntuación media pasa de 4,6 a 2,7 puntos; por lo tanto, de malestar clínicamente moderado-severo a malestar leve. Estas diferencias son aún mayores si la comparación se establece con el estado 
emocional justo antes de la visita, con un promedio de afectación de 17,8 puntos, que pasa a 11,3 después de la visita. Parece ser que el hecho de encontrase en el centro sanitario podría activar o aumentar las emociones negativas vividas a lo largo de la semana previa. En todo caso, después de la visita psicológica, estos valores se reducen significativamente. Además, un elevado porcentaje de mujeres se sienten, después de la visita, animadas, valientes, con interés por las actividades cotidianas, tranquilas y alegres, siendo significativo el número de mujeres que pasa de experimentar el polo negativo de la emoción durante la última semana a experimentar el polo positivo después de la visita. Más de tres cuartas partes de la muestra experimentan 3 o más emociones positivas después de la visita.

También es significativo el número de mujeres que pasan de estar preocupadas a despreocupadas, pero, no obstante, solo un tercio de la muestra aproximadamente se siente despreocupada después de la visita. Parece, por tanto, que la preocupación es el aspecto más difícil de manejar y cambiar mediante la sesión de terapia psicológica. La emoción experimentada con más intensidad antes de la visita es el nerviosismo, seguido por la tristeza, el miedo y la rabia. Después de la visita disminuye significativamente su intensidad, excepto en el caso de la rabia, que disminuye de forma no significativa. Después de la visita la rabia pasa a ser la emoción más intensa seguida por las otras tres, las cuales mantienen el orden inicial entre ellas. Es posible que ello sea debido a diversas razones. Por una parte, la rabia es difícil de expresar en la interacción terapéutica, por lo que puede ser ignorada y no tratada. Por otro lado, las disminuciones pueden ser mayores, lógicamente, en aquellas emociones más intensas al principio y, en este caso, la rabia era de las emociones menos intensas, coin- cidiendo con diversos estudios realizados en nuestro entorno ${ }^{(3,4)}$ que apuntan que la ira y la rabia no suelen ser emociones predominantes entre las pacientes de cáncer de mama.

A partir de las matrices de correlaciones y las relaciones significativas que en éstas se observan, podemos concluir que, cuanto mayor es el malestar experimentado la última semana, mayor es, también, el experimentado después de la visita. Es decir, aquellas pacientes que han experimentado más malestar la última semana siguen siendo las que experimentan más malestar después de la visita. En segundo lugar, la relación significativa existente entre el malestar de la última semana y la intensidad de tristeza antes de la visita no se constata con el resto de emociones. Por tanto, el nivel de malestar experimentado la última semana solo podría predecir, en parte, el nivel de tristeza antes de la visita. En tercer lugar, las intensidades de las distintas emociones antes de la visita correlacionan positivamente entre sí y, también lo hacen las intensidades de las emociones después de la visita. Esto parece indicar que estos estados emocionales guardan la misma relación entre sí, aunque disminuyan significativamente su intensidad, después de la visita psicológica.

Finalmente, variables como la situación médica en la que se encuentran las mujeres y el número de visita, no se relacionan de forma significativa con el estado emocional de la última semana. Es decir, el malestar emocional y el total de problemas emocionales experimentados no difieren de forma significativa según si las pacientes acudían a una primera visita o si ya habían tenido visitas anteriormente $\mathrm{ni}$, tampoco, entre aquellas mujeres que se encontraban en fase de diagnóstico, tratamiento o seguimiento. Éste hecho se constataba también en el estudio de Lester et al. ${ }^{(28)}$, citado anteriormente, encontrando los autores que el nivel de malestar 
emocional no variaba significativamente entre mujeres que se encontraban en distintas fases de la enfermedad, excepto en aquellas que ya habían transcurrido seis meses desde la finalización del tratamiento, las cuales experimentaban un declive del nivel de malestar emocional. En nuestro estudio, las variables comentadas (situación médica y número de visita) tampoco influían de forma significativa en el estado emocional de antes de la visita ni en el cambio emocional que experimentaban las pacientes después de la visita. No obstante se observó que las mujeres que acababan de recibir el diagnóstico experimentaban rabia, miedo y nerviosismo de forma ligeramente más intensa que las pacientes de otras situaciones médicas. De hecho, varios estudios han encontrado que el malestar es más intenso durante la fase de diagnóstico ${ }^{(28)}$, sugiriendo otros que la calidad de vida de las mujeres con cáncer de mama mejora a medida que trascurre el tiempo desde el diagnóstico y el tratamiento ${ }^{(37)}$. En todo caso, parece ser que la fase de diagnóstico es la que suele generar mayor malestar emocional, como se ha constatado en otros estudios $^{(3,4)}$. No obstante, algunos autores destacan la persistencia e incluso aumento de síntomas relacionados con el estrés una vez finalizado el tratamiento ${ }^{(38)}$. En relación al cambio emocional favorable, éste era mayor en aquellas mujeres que se encontraban en la fase de tratamiento o en la fase de seguimiento (intervalo libre) y, en aquellas mujeres que ya habían realizado algunas visitas con la psico-oncóloga previamente. Esto último indicaría que se da un cierto efecto de aprendizaje terapéutico. Así pues, se puede suponer que en términos clínicos la intervención evaluada resulta igual de eficaz sea cual sea la situación médica de las pacientes $y$, se trate de primeras visitas o visitas posteriores, lo que apoyaría la propuesta de tratamientos transdiagnósticos, ya que in- dependientemente de las particularidades de cada situación, se consiguen mejorías similares con un mismo procedimiento.

A partir de estos resultados se puede concluir que una sesión psico-oncológica, en la que se aborda la experiencia global y subjetiva de las pacientes desde el counselling y la psicología positiva, tiene un impacto emocional favorable inmediato. Además de producirse un alivio del malestar emocional experimentado, las pacientes experimentan, después de la sesión, estados emocionales positivos. Estos resultados se complementan con la información cualitativa obtenida por Nekolaichuk et al. ${ }^{(39)}$, sobre los beneficios de una sesión o sesiones iniciales de counselling percibidos por los pacientes: varios de ellos comentaron que habían experimentado una mejora después de solamente una o dos sesiones y consideraron que el encuentro terapéutico (coincidente en gran parte con el que se evalúa en el presente estudio), les había permitido explorar, adaptarse, cambiar, movilizar sus recursos de afrontamiento, ver las cosas desde otra perspectiva y conseguir una cierta sensación de curación, entre otros beneficios. Otros estudios con pacientes de cáncer de mama también han constatado la eficacia y los beneficios de programas con elementos comunes a la terapia utilizada en la presente investigación. Es el caso, por ejemplo, de programas de counselling individual o entre iguales ${ }^{(40)} y$, también, vía telefónica ${ }^{(41,42)}$; o el programa para la Reducción del Estrés Basado en la Atención Plena ${ }^{(10,38,43-46)}$. Relacionado con ello, Greenlee et al. ${ }^{(46)}$, en su revisión sistemática concluyen que la meditación, el yoga y la relajación con imaginación son muy recomendables para las pacientes con cáncer de mama.

Somos conscientes de las limitaciones de la presente investigación por lo que los datos obtenidos deben considerarse como exploratorios y preliminares para futuras investigaciones. En primer lugar el tamaño 
de la muestra es reducido. En segundo lugar, aunque los datos muestran claramente la mejora tras la sesión, este estudio no permite conocer qué elementos o procedimientos produjeron determinados efectos sobre qué individuo. Sin embargo, como se ha comentado anteriormente, éste no era el objetivo del presente estudio. Sería aconsejable que estudios futuros controlen o incluyan medidas más específicas sobre las diferentes variables implicadas. En nuestro caso, la renuncia a ciertas medidas y cuestionarios estandarizados, válidos y fiables, responde a distintas cuestiones: 1) inexistencia de un cuestionario que se adaptara al objetivo del presente estudio, es decir, la valoración del cambio emocional en una sola sesión; 2) intención de obtener, con la mayor brevedad y rapidez posible, una información básica sobre el estado emocional; 3) utilizar pocos instrumentos y breves, evitando preguntas largas $y$, rehuyendo incidir en matices repetitivos y alargar el tiempo requerido para no generar malestar o inducir ciertas cogniciones y/o reacciones emocionales. Por otro lado, no considerar la presencia de patologías psicológicas concretas como un criterio de tratamiento diferenciado se basa en un enfoque transdiagnóstico, entendiendo que la experiencia de padecer un cáncer acarrea un coste emocional en todas las mujeres, independientemente de su situación de partida. Se pretende entender pues, el sufrimiento humano como un todo ${ }^{(18)}$. Además, el funcionamiento psicológico, social y físico puede predecirse más allá de los niveles de trastornos psicopatológicos, teniendo en cuenta el despliegue de componentes propios de una salud mental positiva, como la satisfacción con la vida o los propósitos en ésta ${ }^{(16)}$. Por último, sería necesario investigar cómo evoluciona este cambio inmediato a lo largo de las distintas sesiones y, también, la consolidación y mantenimiento de los efectos a corto y largo plazo. Así mismo, sería interesante abarcar pacientes con otros tipos de cáncer y terapias con orientaciones y formatos distintos.

A nivel práctico, a pesar de las limitaciones indicadas, este estudio informa sobre las dificultades emocionales que pueden atravesar las mujeres con cáncer de mama y la importancia de integrar en la cura rutinaria de éstas el tratamiento psicológico, potenciando su derivación al servicio de psico-oncología, habiéndose constatado un alivio emocional tras discutir y abordar aspectos importantes para ellas. Tras una primera valoración se deberá decidir si es necesario o no un seguimiento para acompañar y ayudar a la paciente a afrontar la enfermedad de una manera adaptativa. Además, deben tenerse en cuenta los beneficios clínicos que puede tener una sola sesión y la importancia que este último hecho tiene en términos de brevedad y economía. Finalmente, pone de relieve el peso de la relación terapéutica más allá de las técnicas empleadas. Todos estos datos pueden ser útiles para aumentar el conocimiento de los profesionales, implicados en el tratamiento de las pacientes, acerca de los efectos positivos de la intervención psicológica, favoreciendo el trabajo en equipo y repercutiendo, en último término, a ofrecer una atención global y de calidad a estas pacientes.

\section{AGRADECIMIENTOS}

Agradecemos a las pacientes y los profesionales del Hospital Comarcal Sant Jaume de Calella su participación en este estudio.

\section{REFERENCIAS BIBLIOGRÁFICAS}

1. Sociedad Española de Oncología Médica. Las Cifras del Cáncer en España 2016. [Internet]. Madrid: Sociedad Española de Oncología Médica; 2016 [citado 12 Feb 2016]. Disponible en: http://seom.org/ 
seomcms/images/stories/recursos/LA_ClFRAS_DEL_CANCER_EN_2016.pdf

2. Bellver A, Sánchez-Cánovas J, Santaballa A, Munárriz B, Pérez-Fidalgo JA, Montalar J. Mujeres con cáncer de mama: Evaluación del afecto positivo y negativo y valoración de un programa de intervención psicológica en el ámbito hospitalario. Psicooncologia 2009;6:139-54.

3. Font A, Rodríguez E. Eficacia de las intervenciones psicológicas en mujeres con cáncer de mama. Psicooncologia 2007;4:423-46.

4. Font A, Rodríguez E. Eficacia de la terapia de grupo en cáncer de mama: variaciones en la calidad de vida. Psicooncologia 2004;1:67-86.

5. Font A, Cardoso A. Afrontamiento en cáncer de mama: pensamientos, conductas y reacciones emocionales. Psicooncologia 2009;6:27-42.

6. Holland JC, Andersen B, Breitbart WS, Buchmann LO, Compas B, Deshields TL, et al. NCCN Clincal Practice Guidelines in Oncology. Distress management. Version 2.2013 [Internet]. National Comprehensive Cancer Network; 2013 [citado el 12 Feb 2016]. Disponible en: http:// oralcancerfoundation.org/treatment/pdf/ distress.pdf

7. Yélamos C, Montesinos F, Eguino A, Fernández $B$, González A, Paredes MG De. "Mucho x Vivir". Atención psicosocial para mujeres con cáncer de mama. Psicooncologia. 2007;4:417-22.

8. Ganz PA, Kwan L, Stanton AL, Bower JE, Belin TR. Physical and psychosocial recovery in the year after primary treatment of breast cancer. J Clin Oncol. 2011;29:11019. Doi:10.1200/JCO.2010.28.8043

9. Vázquez C, Castilla C. Emociones positivas y crecimiento postraumático en el cáncer de mama. Psicooncologia 2007;4:385-404.

10. Casellas-Grau A, Font A, Vives J. Positive psychology interventions in breast cancer. A systematic review. Psychooncology 2014;(23):9-19. doi:10.1002/pon.3353
11. Fuentes $\mathrm{S}$, Blasco T. Factores predictores de la adaptación a la enfermedad en pacientes recién diagnosticadas de cáncer de mama que acuden a un servicio de psicooncología. An Psicol 2012;28:73642. Doi:10.6018/analesps.28.3.156031

12. Fernández J, Bárez M, Blasco T. La inducción de sensación de control como elemento fundamental de la eficacia de las terapias psicológicas en pacientes de cáncer. An Psicol 2003;19:235-46.

13. Vázquez C, Hervás G, Ho SMY. Intervenciones clínicas basadas en la Psicología Positiva: Fundamentos y aplicaciones. Psicol Conductual 2006;14:401-32.

14. Vázquez C, Hervás G, Rahona JJ, Gómez D. Bienestar psicológico y salud: Aportaciones desde la Psicología Positiva. Anu Psicol Clín Salud 2009;5:15-28.

15. Seligman M. Flourish: Positive Psychology and Positive Interventions. The Tanner Lectures on Human Values, 2010.

16. Gallagher MW, Sauer-Zavala SE, Boswell JF, Carl JR, Farchione TJ, Barlow DH. The impact of the Unified Protocol for Emotional Disorders on quality of Life. Int J Cogn Ther. 2013;6:1-16. Doi:10.1521/ ijct.2013.6.1.57

17. Fredrickson BL. The broaden-and-build theory of positive emotions. Phil Trans R Soc Lond B. 2004;359:1367-77. Doi:10.1098/rstb.2004.1512

18. Belloch A. Propuestas para un enfoque transdiagnóstico de los trastornos mentales y del comportamiento: evidencia, utilidad y limitaciones. Rev Psicopatología y Psicol Clín 2012;17:295-311.

19. Remor E, Arranz P, Ulla S. El psicólogo en el ámbito hospitalario. Bilbao: Desclée De Brouwer; 2003.

20. Shiffman S, Stone AA, Hufford MR. Ecological Momentary Assessment. Annu Rev Clin Psychol. 2008;4:1-32. Doi:10.1146/ annurev.clinpsy.3.022806.091415

21. Badr H, Pasipanodya EC, Laurenceau J-P. An electronic diary study of the effects of patient avoidance and partner social 
constraints on patient momentary affect in metastatic breast cancer. Ann Behav Med 2013;45:192-202. Doi: 10.1007/s12160012-9436-8

22. Ratcliff CG, Lam CY, Arun B, Valero V, Cohen L. Ecological momentary assessment of sleep, symptoms, and mood during chemotherapy for breast cancer. Psychooncology 2014;23:1220-8. Doi:10.1002/pon.3525

23. Banthia R, Malcarne VL, Ko CM, Varni JW, Sadler GR. Fatigued breast cancer survivors: The role of sleep quality, depressed mood, stage and age. Psychol Health 2009;24(8):965-80. doi:10.1080/08870440802110831

24. Maté J, Sirgo A, Mateo D, Bayés R, Bernaus $M$, Casas $C$, et al. Elaboración y propuesta de un instrumento para la detección de malestar emocional en enfermos al final de la vida. Psicooncologia 2009;6:507-18.

25. Sohl S, Schnur J, Sucala M, David D, Winkel G, Montgomery G. Distress and emotional well-being in breast cancer patients prior to radiotherapy: An expectancy-based model. Psychol Health 2012;27:347-61. Doi: 10.1080/08870446.2011.569714

26. Miller S, Schnur J, Weinberger-Litman, Sarah, Montgomery G. The relationship between body image, age, and distress in woman facing breast cancer surgery. Palliat Support Care 2014;12:363-7. Doi:10.1017/S1478951513000321

27. Graves KD, Schmidt JE, Bollmer F, Fejfar M, Langer S, Blonder LX, et al. Emotional expression and emotional recognition in breast cancer survivors: A controlled comparison. Psychol Health 2005;20:579-95. Doi:10.1080/0887044042000334742

28. Lester J, Crosthwaite K, Stout R, Jones R, Holloman C, Shapiro C, et al. Women with breast cancer: Self-reported distress in early survivorship. Oncol Nurs Forum 2015;42:554-61. Doi:10.1188/15.ONF. E17-E23

29. Bultz BD, Holland JC. Emotional distress in patients with cancer: the sixth vital sign. Community Oncol 2006;3:311-4.
30. Hernández M, Cruzado JA, Prado C, Rodríguez E, Hernández C, González MA, et al. Salud mental y malestar emocional en pacientes con cáncer. Psicooncologia 2012;9:233-57. Doi:10.5209/ rev_PSIC.2013.v9.n2-3.40895

31. Mosher C, Johnson C, Dickler M, Norton L, Massie M.J, DuHamel K. Living with metastatic breast cancer: A qualitative analysis of physical, psychological, and social sequelae. Breast J 2013;19:285-92. Doi:10.1111/tbj.12107

32. Limonero JT, Mateo D, Maté-Méndez J, González-Barboteo J, Bayés R, Bernaus $M$, et al. Evaluación de las propiedades psicométricas del cuestionario de Detección de Malestar Emocional (DME) en pacientes oncológicos. Gac Sanit;2012;26:145-52. Doi: 10.1016/j. gaceta.2011.07.016

33. Hernández M, Cruzado JA. La atención psicológica a pacientes con cáncer: de la evaluación al tratamiento. Clín Salud 2013;24:1-9. Doi:10.5093/cl2013a1

34. Almanza-Muñoz JDJ, Holland JC. Psicooncología: estado actual y perspectivas futuras. Rev Inst Nac Cancerol (Mex) 2000;46:196-206.

35. Moscoso M, Knapp M. La necesidad de evaluar distrés emocional en psicooncología: ciencia o ficción? Rev Psicol 2010;28:285-309.

36. Montero I, León OG. A guide for naming research studies in Psychology. Int J Clin Heal Psychol 2007;7(3):847-62.

37. Janz NK, Friese CR, Li Y, Graff JJ, Hamilton AS, Hawley ST. Emotional well-being years post-treatment for breast cancer: Prospective, multi-ethnic, and population-based analysis. J Cancer Surviv 2014;8:131-42. Doi:10.1007/s11764013-0309-3

38. Charlson ME, Loizzo J, Moadel A, Neale M, Newman C, Olivo E, et al. Contemplative self healing in women breast cancer survivors: a pilot study in underserved minority women shows improvement in 
quality of life and reduced stress. BMC Complement Altern Med 2014;14:1-10. Doi:10.1186/1472-6882-14-349

39. Nekolaichuk CL, Turner J, Collie K, Cumming C, Stevenson A. Cancer patients' experiences of the early phase of individual counseling in an outpatient psycho-oncology setting. Qual Health Res 2012;23:592604. Doi:10.1177/1049732312470567

40. Ramsay K, Ramsay J, Main D. Both group peer counselling and individual counselling reduce anxiety and depression, and increase self-esteem and overall life satisfaction in palliative cancer care. Couns Psychol Q 2007;20:157-67. Doi:10.1080/09515070701240071

41. Garrett K, Okuyama S, Jones W, Barnes D, Tran Z, Spencer L, et al. Bridging the transition from cancer patient to survivor: Pilot study results of the Cancer Survivor Telephone Education and Personal Support (C-STEPS) program. Patient Educ Couns 2013;92:266-72. Doi:10.1016/j. pec.2013.04.002

42. Marcus AC, Garrett KM, Cella D, Wenzel L, Brady MJ, Fairclough D, et al. Can telephone counseling post-treatment improve psychosocial outcomes among early stage breast cancer survivors? Psychooncology 2010;19:923-32. Doi:10.1002/ pon. 1653
43. Eyles C, Leydon GM, Hoffman CJ, Copson ER, Prescott P, Chorozoglou M, et al. Mindfulness for the Self-Management of Fatigue, Anxiety, and Depression in Women With Metastatic Breast Cancer A Mixed Methods Feasibility Study. Integr Cancer Ther 2014;14:42-56. Doi:10.1177/1534735414546567

44. Lengacher $C A$, Shelton MM, Reich $R$, Barta B, Johnson-Mallard V, Moscoso M, et al. Mindfulness based stress reduction $(\mathrm{MBSR}(\mathrm{BC}))$ in breast cancer: evaluating fear of recurrence (FOR) as a mediator of psychological and physical symptoms in a randomized control trial (RCT). J Behav Med 2014;37:185-95. Doi:10.1007/ s10865-012-9473-6

45. Henderson VP, Massion AO, Clemow L, Thomas G, Druker S, Hébert JR. A randomized controlled trial of mindfulnessbased stress reduction for woman with early-stage breast cancer receiving radiotherapy. Integr Cancer Ther 2013;12:40413. Doi:10.1177/1534735412473640

46. Greenlee H, Balneaves LG, Carlson LE, Cohen M, Deng G, Hershman D, et al. Clinical Practice Guidelines on the Use of Integrative Therapies as Supportive Care in Patients Treated for Breast Cancer. J Natl Cancer Inst Monogr 2014;50:346-58. Doi:10.1093/jncimonograhps/lgu041 
